\title{
Una Revisión de la Interpretación Económica sobre la Innovación
}

\author{
Luis Miguel Beristain Hernández'
}

\begin{abstract}
Theorists have employed two conflicting approaches in order to understand the economic concept of innovation: the classical theory of static equilibrium where innovation is interpreted as being exogenous to the economic system, and the dynamic equilibrium theory of endogenous technological change. Despite progress in studies and inquiries on the second model, research in and teaching of economics remain dominated by the first approach. This review discusses the differences between science, technology and innovation and implications of dynamic equilibrium theory in understanding the economy and wealth creation processes.
\end{abstract}

Keywords: innovation, technological change, static equilibrium, dynamic equilibrium.

\section{Resumen}

El concepto económico de la innovación ha sido tratado desde dos enfoques antagónicos: el de la teoría clásica del equilibrio estático donde la innovación es interpretada como un elemento exógeno al sistema económico, y el de la teoría del equilibrio dinámico del cambio tecnológico endógeno. A pesar del avance en los estudios del segundo modelo, la investigación y la enseñanza de la economía continúa siendo dominada por el primer enfoque. En la presente revisión se discute las diferencias entre ciencia, tecnología e innovación, y las implicaciones de la teoría del equilibrio dinámico para entender la economía y los procesos de creación de riqueza.

Palabras clave: innovación, cambio tecnológico, equilibrio estático, equilibrio dinámico.

\footnotetext{
' Instituto Tecnológico y de Estudios Superiores de Monterrey, Campus Ciudad de México. Calle del Puente 222, Col. Ejidos de Huipulco. México, D.F. 14380. Teléfono: +52 5554832390 / Fax: +52 555483 2082. E-mail: Lberista@itesm.mx
}

ISSN: 07 I8-2724. (http://www.jotmi.org)

Journal of Technology Management \& Innovation ( Universidad Alberto Hurtado, Facultad de Economía y Negocios 


\section{Introducción}

Diferentes disciplinas y ámbitos de estudio han ofrecido su explicación sobre el concepto de innovación. En el presente trabajo se brinda una revisión de la literatura relevante de la ciencia económica para su entendimiento. Sin pretender ser exhaustivo ni agotar las referencias, se procede a identificar las escuelas y los enfoques principales contrastando con la definición neoclásica. Otros autores han abordado el fenómeno desde la sociología, el concepto evolucionista de la tecnología y de los mercados, la psicología, los estudios organizacionales y por supuesto la literatura administrativa, los cuales han de ser abordados en entregas posteriores.

Aún cuando la innovación es uno de los fenómenos más relevantes para la sociedad, y debiera constituir uno de los ejes de la política pública y de la práctica empresarial, recibe una atención inadecuada debido al poco entendimiento sobre el tema. La revisión aquí presentada ayudará a construir una plataforma de entendimiento común a partir de las principales fuentes de controversia: la primera, la distinción entre ciencia, tecnología e innovación como fenómenos relacionados pero de naturaleza distinta. La segunda, el debate entre la teoría clásica del equilibrio estático a partir de la relación entre los retornos decrecientes de la producción y el principio de la utilidad marginal del consumo, y el efecto desestabilizador del cambio tecnológico y la innovación. Sin pretender dar una respuesta satisfactoria se explorará el planteamiento de las diferentes corrientes para discutir estos temas.

\section{La innovación en la disciplina económica}

Si bien los distintos enfoques coinciden en definir la ciencia económica como el estudio del comportamiento humano en el proceso de elección en condiciones de recursos con usos alternativos y limitados con relación a los deseos (Coase, 1994), pueden distinguirse al menos dos posiciones contradictorias entre sí. La primera de ellas, y conocida como la teoría neoclásica, se construye a partir de la noción de equilibrio entre la oferta y la demanda, donde la producción infinita $\circ$ la satisfacción de necesidades y deseos también de manera creciente, sencillamente sería un absurdo, por lo tanto el mercado tiende al equilibrio por motivos de los retornos decrecientes de la producción y la utilidad marginal también decreciente del consumo, relación traducida en información a través del precio (Beinhocker, 2006). En esta concepción los cambios en las cantidades, específicamente el crecimiento económico, será producto de los cambios en la población y el capital, pero también de factores exógenos como los fenómenos naturales, las guerras, el clima y las invenciones, todas ellas variables aleatorias en la ecuación (Schumpeter, 2002). En consecuencia, el cambio tecnológico y la innovación son considerados fenómenos externos al sistema económico. La segunda de ellas, la cual podríamos denominar del "desarrollo en progreso" o la escuela positiva del cambio tecnológico, interpreta la economía como un sistema de equilibrio inestable o de equilibrio dinámico, donde la innovación es considerada una variable endógena y propia del sistema (Romer, 1986). A continuación se ha de revisar la literatura que aborda la innovación como un componente endógeno y propio del conjunto económico, dándole un carácter originario para afectar las condiciones de equilibrio estático y crear la riqueza material necesaria para satisfacer los deseos y las necesidades humanas a partir de recursos escasos, los cuales son transferidos constantemente a actividades de mayor rendimiento y productividad.

\section{La teoría positiva del cambio tecnológico}

La noción básica de la innovación, entendida como la acción de dotar a los recursos con nuevas posibilidades de producir riqueza, es expuesta por Jean-Baptiste Say en el año de 1803. Con toda claridad se refiere a la actividad económica como el espacio donde el valor es literalmente producido por la acción creadora del trabajo: "Los objetos... no pueden ser creados por ningún medio humano; ni la masa de la materia... incrementada o disminuida. Todo lo que el hombre puede hacer, es reproducirla en otra forma..." (2002, p. 26). Si el ser humano no puede crear la materia, sí puede crear su utilidad, aumentarla o disminuirla, y en ello consiste la innovación. Posiblemente sea el primero de los pensadores en reconocer la importancia de la "inteligencia y el conocimiento de las leyes de la naturaleza” (2002, p. 3I) para dominarla y obtener el mayor valor posible de ella a través de invenciones como la maquinaria, por emplear el lenguaje de la época, convirtiéndole en un motor de creación de riqueza. El avance científico o la acumulación de conocimiento no son condición suficiente para dicha creación, sino "la hermosa habilidad práctica de sus emprendedores para aplicar útilmente el conocimiento y la

ISSN: 07I 8-2724. (http://www.jotmi.org)

Journal of Technology Management \& Innovation @ Universidad Alberto Hurtado, Facultad de Economía y Negocios 
habilidad superior de sus trabajadores para ejecutarla rápida y magistralmente.” (2002, p. 34).

Ciento veinticinco años después Joseph Schumpeter, en una clara muestra de inconformidad con la explicación de la teoría clásica del equilibrio, definiría la innovación "como aquellos cambios en la combinación de los factores de producción que no pueden ser afectados por infinitesimales pasos o variaciones en el margen" (2002, p. 30). Contraria a la interpretación exógena de la innovación en la tradición del pensamiento de la época, para Schumpeter es una variable endógena y propia del sistema económico expresado en la forma de nuevos productos, de nuevos métodos de producción, de nuevos mercados, de la utilización de nuevos insumos o en el diseño de nuevas formas de organización en una industria específica (Schumpeter, 2004). El agente central en su teoría, como en Say, es la acción emprendedora motivada por la búsqueda de las utilidades temporales resultantes de explotar de manera distinta las posibilidades tecnológicas de una invención (Schumpeter, 1975). Esta acción en sus primeros escritos pertenecía al emprendedor como creador de la nueva organización productiva, mientras en su última obra, a la "función emprendedora" desde el interior de la organización empresarial establecida.

Los estudios del crecimiento o del desarrollo económico inaugurados por Schumpeter han considerado la innovación como una fuerza desequilibrante del sistema económico (Brunn, 1974; Ashton, 1975; Laski, 1977; Bartel y Sicherman, 1999), distinguiendo claramente el avance científico de la invención técnica y éstos de la innovación propiamente dicha (Laski, 1977; More, 2000; Aghion, et al., 2002; Acemoglu y Linn, 2003). La primera es la búsqueda sistemática de explicaciones generales a un fenómeno (Ordóñez, 200I) en tanto la invención técnica es "una solución original resultado de la síntesis de información acerca de las necesidades o deseos, e información acerca de los medios técnicos por los cuales se pueden satisfacer" (Utterback, 197I, p. 77). "Sin embargo, la tecnología no es sirviente de la ciencia” (Basalla, 1999, p. 27), una y otra se influyen mutuamente: el motor de vapor se inventó y aplicó antes de contar con una teoría de la termodinámica; de hecho el primero motivó el desarrollo de la segunda (Lienhard, 2006). Por su parte la innovación es un fenómeno enteramente económico de creación de riqueza: "Un proceso que toma una idea o invención, la relaciona con una demanda de mercado y le convierte en un producto, una técnica o un servicio que es comprado y vendido..." (Bas, et al., 2008, p. 53).

En este paradigma las variables que explican la innovación se concentran en la búsqueda de los incentivos económicos de mercado -utilidades emprendedoras- o en la reducción de los costos de producción (Nelson, 1959; Brunn, 1974; More, 2000; Rosenberg 2000; Aghion, et al., 2002; Boldrin y Levine, 2002), en los esfuerzos intencionales de investigación y desarrollo (Nelson, 1959; Bartel y Sicherman, 1999; Aghion, et al., 2002;), en la acumulación de capital humano (Ashton, 1975; Lucas, 1993; Bartel y Sicherman, 1999; Teece, 2000), en la explotación de nuevas invenciones o en la aplicación de tecnologías maduras a nuevos usos (Brunn, 1974; Ashton, 1975; More, 2000; Boldrin y Levine, 2002).

En 1956, Robert Solow sugirió cómo los cambios tecnológicos habían afectado dramáticamente la productividad del capital y por lo tanto no podía sostenerse "el supuesto crucial por el cual la producción tiene lugar bajo condiciones de proporciones fijas." (1956, p. 65). A diferencia de Schumpeter, buscó una solución compatible con la interpretación neoclásica al proponer un equilibrio dinámico o un crecimiento balanceado en el cual "el cambio tecnológico sencillamente multiplica la función de producción por un factor de escala creciente" (Solow, p. 85). Sin embargo el cambio tecnológico en su teoría es considerado exógeno, lo cual no satisfizo las inquietudes de Paul Romer, quien a su vez propuso un modelo "de equilibrio de cambio tecnológico endógeno en el cual el crecimiento en el largo plazo es impulsado primariamente por la acumulación de conocimiento" (1986, p. 1003). Como en Say, la materia prima no puede ser cambiada o alterada, "pero como resultado de la prueba y el error, la experimentación, el refinamiento y la investigación científica, las instrucciones que seguimos para combinar las materias primas se convierten más sofisticadas" (1990, p. 72), en eso consiste la innovación. Las variables que explican la innovación son la producción, la transferencia, la acumulación y el uso del conocimiento motivado por los incentivos de mercado, pero a diferencia de los modelos de la economía neoclásica, "donde el capital exhibe retornos marginales decrecientes, el conocimiento puede expandirse sin límite alguno", exhibiendo retornos marginales crecientes (Romer, 1986, p. 1003), lo cual resulta incompatible con la noción clásica del equilibrio estático.

ISSN: 07I 8-2724. (http://www.jotmi.org)

Journal of Technology Management \& Innovation (C) Universidad Alberto Hurtado, Facultad de Economía y Negocios 


\section{La competencia de mercado}

Si bien la escuela del cambio tecnológico aborda el estudio de la creación de riqueza desde el nivel de análisis "macro", donde la innovación es el acto propio de dicha creación, es en el siguiente nivel, el de mercado, donde ocurren las decisiones de producción y de adopción de nuevos productos y de nuevos procesos. Para el modelo neoclásico del equilibrio las empresas proveen los bienes y servicios en función del costo de producción y de la maximización de utilidades, enfrentando restricciones tecnológicas -entendidas éstas como las combinaciones únicas disponibles para las proporciones fijas de capital y de producción- y la curva de demanda para dicho mercado. En un ambiente de competencia pura la empresa sólo puede tomar decisiones sobre la cantidad a producir, actuando como un "tomador" de precio. Cuando la estructura de mercado es de una sola empresa, el monopolio, puede entonces decidir el precio y la cantidad a producir que maximice las ganancias totales. Sin embargo, muchos de los mercados en realidad se definen por un número reducido de competidores, cada uno de ellos con la posibilidad de influir en la cantidad y en el precio, caracterizado por estrategias tales como líderseguidor de precio o líder-seguidor de cantidad. El supuesto básico del modelo se encuentra en la "homogeneidad" del producto y las condiciones estáticas del mercado (Varian, 2003).

Pero las economías de mercado se caracterizan por someter a las organizaciones productivas a presiones competitivas, entendiéndose por tal la interacción de los "esfuerzos realizados para desempeñarse mejor que otros [en cuestiones como] los precios, la calidad y el progreso técnico." (Demsetz, 1997, p. 137). Cuando dicho esfuerzo se orienta principalmente a la calidad y a las cantidades del producto, se dice que la empresa responde con un comportamiento adaptador, sugerido por Schumpeter (2002) y operacionalizado por Kirton (2003) como aquella inclinación estable en el tiempo para buscar mejores y más eficientes formas de hacer las cosas, en este caso la producción. Pero existe un segundo tipo de respuesta la cual consiste en ofrecer una propuesta de valor completamente distinta a la existente, escapando con ello de la competencia de mercado; Schumpeter le llamó comportamiento innovador: las personas $y$ las organizaciones "pueden reaccionar haciendo nuevas cosas o las mismas cosas de una manera diferente, incompatible con el arreglo fundamental existente" (Schumpeter, 2002, p. 27). En consecuencia, la competencia no se define en términos de la homogeneidad del producto, sino por el grado de sustitución entre éstos (Baggs y de Bettignies, 2006): con seguridad los agentes de la industria azucarera en México hubieran diseñado estrategias distintas en la década de los 90 s de haber previsto la importancia de la alta fructosa de maíz en la producción de bebidas carbonatadas y no limitarse a considerar únicamente el impacto de la oferta australiana o brasileña de caña de azúcar, como con seguridad ocurrió. Siguiendo el argumento, la innovación es entonces una respuesta específica de diferenciación y de naturaleza distinta a la calidad y al costo, cuyo resultado es el escape de las presiones de la competencia. Más importante aún, ante la ausencia de presión competitiva no existen los incentivos de mercado para fomentar la innovación y el cambio tecnológico (Demsetz, 1997).

Ahora bien, la intensidad de la competencia en su máxima expresión ocurrirá en mercados desconcentrados caracterizados por un gran número de empresas con productos idénticos, donde ninguno de los participantes puede ejercer control alguno sobre el mercado (Demsetz, 1997). Pero cumplir con las premisas de atomicidad, de homogeneidad, de perfección de información, de igualdad de acceso a la tecnología y el conocimiento relevante, así como la libertad de entrada y de salida, pareciera una ingenuidad (Teece, 2000). Dadas éstas restricciones, la intensidad de la competencia será resultado del número de organizaciones y de la fuerza en las respuestas individuales, de donde deriva la hipótesis según la cual las grandes empresas estarán mejor dotadas para competir en términos de volumen, de costo y de calidad en los espacios de competencia imperfecta (Mansfield, 1963; Williamson, 1975; Romo y Hill de Titto, 2006). Sin embargo la estructura de la industria, sea un monopolio, un oligopolio, un monopsonio $\circ$ un oligopsonio, pierde importancia cuando se introduce una innovación: "Así, la competencia innovadora, o como Schumpeter la describe, la destrucción creativa, funciona a través de cambios en la tecnología, en la organización, en el producto, y opera en mercados monopólicos en el sentido estándar... [en donde] un pequeño grupo de individuos crean nuevos mercados o revolucionan uno viejo. Las personas actúan como si pudieran influir en los resultados, y de hecho así lo hacen" (Demsetz, 1997, p. 138).

ISSN: 07I 8-2724. (http://www.jotmi.org)

Journal of Technology Management \& Innovation (C) Universidad Alberto Hurtado, Facultad de Economía y Negocios 
Aún cuando diversos estudios relacionados con la competencia de mercado coinciden en referir la innovación como una estrategia específica para escapar de las presiones competitivas, los resultados parecieran ser inconsistentes al considerar el tamaño de la empresa y la concentración del mercado (Geroski, 1989; Henderson, 1993; Koeller, 1995; Blundell, et al., 1999; Porter, 1999): en algunos casos se ha encontrado evidencia sobre cómo las innovaciones favorecen la desconcentración de la estructura industrial, particularmente cuando la innovación es introducida por una compañía pequeña o por un nuevo competidor (Geroski y Pomroy, 1990), e identificándose una relación inversa entre el grado de concentración y el número de innovaciones observadas (Koeller, 1995); pero en otros la intensidad se explica por la introducción de nuevas soluciones como resultado del esfuerzo realizado por grandes organizaciones en sectores altamente concentrados con el control necesario sobre el mercado y la holgura de recursos como para beneficiarse de la innovación (Mansfield, 1963; Henderson, 1993). A manera de explicar estas aparentes contradicciones, se puede establecer que las innovaciones sostenibles e incrementales suelen ser introducidas exitosamente por la gran empresa establecida, mientras las innovaciones de ruptura, por el contrario, se relacionan con la organización emprendedora y con la empresa invasora -aquella establecida en otros mercados con una propuesta transferible al espacio competitivo relevante (Utterback, 1996; Christensen, 2005). Finalmente, el tamaño de la organización pareciera relacionarse inversamente al considerar la rentabilidad de la innovación (Gopalakrishnan, 2000). En todo caso, los resultados de los diversos estudios soportan la conclusión según la cual la actividad innovadora de las grandes y pequeñas empresas responden a regímenes distintos de tecnología (Acs y Audretsch, 1988) y a la capacidad de introducir cambios exitosos para mejorar el control sobre el mercado y la participación en el mismo, independientemente del tamaño del sistema cooperativo término que se empleará a lo largo del presente trabajo como sinónimo de empresa o de organización (Mansfield, 1963; Blundell, et al., 1999; Boldrin y Levine, 2003).

Así considerada, la innovación es afectada en orientación e intensidad por la concentración de la industria, por el costo de desarrollo e introducción, y por el tamaño del mercado presente o potencial, señal de las oportunidades de obtención de beneficios o ganancias (Scherer, 1982; Koeller, 1995; Boldrin y Levine, 2003;). En general, los diversos autores consideran como innovación sólo aquella basada en el conocimiento científico y en el desarrollo tecnológico, por consecuencia, la definición operacional se expresa en términos del número de patentes (Mansfield, 1963; Scherer, 1982; Koeller, 1995; Blundell, et al., 1999) y en la participación de mercado capturada como resultado de su utilidad económica (Geroski y Pomroy, 1990; Robinson, 1990; Henderson, 1993; Afuah, 2002). Así conceptualizadas, las variables independientes tienen que ver con la intensidad o la inversión en investigación y desarrollo (Mansfield, 1963; Koeller, 1995; Gatti, 1998); con la disposición de capital humano según la proporción de científicos e ingenieros con relación al personal de la empresa, así como con el número de trabajadores con la habilidad para ejecutar los cambios o aprender nuevas competencias (Koeller, 1995; Gatti, 1998; Afuah, 2002). De igual importancia resultan la presencia de recursos complementarios distintos al capital humano y de conocimiento para introducir la innovación al mercado (Gatti, 1998; Boldrin y Levine, 2003), y la intensidad de la inversión en mercadotecnia para favorecer la difusión del nuevo producto (Koeller, 1995; Blundell, et al., 1999).

En todo caso, la perspectiva sobre la competencia interpreta la innovación como una entre varias estrategias para mejorar el desempeño de la empresa en el espacio competitivo, reduciendo la "sustituibilidad" del producto con relación a la oferta disponible en el mercado (Demsetz, 1997; Baggs y de Bettignies, 2006), siendo la actividad innovadora una tarea de rutina para ciertas industrias, mientras para otras, representa una verdadera opción estratégica (Koeller, 2005), en la que los resultados de la empresa serán una función de los cambios en los insumos de la innovación. Como lo sugiere Zawislak, "Independientemente de la estrategia elegida, el propósito principal de las empresas es dominar el medio ambiente, la cadena de valor, las competencias y las capacidades, esto es, sostener el crecimiento de las rutas propias de desarrollo tecnológico" (Zawislak y Marins, 2007, p. 46).

\section{La teoría de los costos de transacción}

Los modelos del cambio tecnológico y de la competencia de mercado dan por hecho la existencia de la empresa, a fin de cuentas el agente principal en la producción de los bienes y la introducción de los cambios. Pero para la escuela de los costos de transacción, su existencia requiere una justificación: ¿si el mercado es eficiente, por qué

ISSN: 07I 8-2724. (http://www.jotmi.org)

Journal of Technology Management \& Innovation (c) Universidad Alberto Hurtado, Facultad de Economía y Negocios 
organizar las actividades del sistema económico en una jerarquía? La respuesta la ofreció Coase al sugerir cómo la empresa no es sino el mecanismo de coordinación alterno al mecanismo de precios "para evitar los costos de llevar a cabo las transacciones en el mercado" (1994, p. 8), transfiriendo la coordinación de los factores a la decisión administrativa ante circunstancias de "racionalidad limitada y de oportunismo" (Williamson, 1985, p. 47). Así entendida, la innovación no sólo hace referencia al cambio en la tecnología del producto, sino también a la introducción de nuevas tecnologías de proceso, de nuevas rutinas - administrativas o productivas $-\mathrm{y}$ de nuevos arreglos jerárquicos, alterando tanto los costos de organización como los costos de usar el mecanismo de precios. En todo caso, el efecto relativo entre ambos costos reducirá el tamaño de la empresa -si se reducen proporcionalmente los costos del mecanismo de precios, o por el contrario, la aumentará si se reducen relativamente los costos de la coordinación administrativa (Coase, 1988).

En consecuencia, la introducción de nuevos productos y de nuevos procesos estará en función no exclusivamente de los incentivos para reducir los costos del mecanismo de precios o los costos de administración, sino de la capacidad de apropiación de los beneficios de la innovación (Williamson, 1965; Teece, 1986; Teece, 2000; Boldrin y Levine, 2003; Moser, 2003). Y por apropiación debe entenderse la capacidad no sólo de proteger a través del régimen de patentes o del secreto comercial el desarrollo, sino de poder aprovecharlo comercialmente (Teece, 1986; Teece, 2000; Moser, 2003) a lo largo de un proceso el cual "incluye no sólo la invención, sino también el desarrollo experimental, las pruebas de mercado, la protección, la producción y la distribución" (Williamson, 1975, p. 197). En consecuencia, es la organización empresarial y no el emprendedor -como en su momento lo argumentaron Say y Schumpeter en su obra temprana-, el agente en condiciones de innovar (Williamson, 1965, 1975, 1995).

Por supuesto, el grado de riesgo y el costo involucrado en el desarrollo, así como los costos administrativos de la replicación y la difusión, determinarán cuáles actividades serán coordinadas en el mercado y cuáles lo serán hacia el interior de la organización. $Y$ aún cuando la introducción de una innovación puede ser realizada por una pequeña organización emprendedora, "la gran empresa podrá al menos emplear los insumos de la misma manera y obtener los mismos beneficios que la primera" (Williamson, 1995, p. 23I), como lo demostró IBM en la exitosa difusión de la computadora electrónica y de la computadora personal a pesar de no ser el introductor de las tecnologías. Más aún, no se trata únicamente de una decisión de costos sino de conveniencia estratégica: en ciertas circunstancias será necesario controlar y proteger la innovación desde la coordinación administrativa (Ogden y Teece, 1980) y en otras se podrá orquestar el desarrollo y la producción a través de los mecanismos de mercado (Andrew y Sirkin, 2006), siendo el caso de la industria farmacéutica ejemplo del primero y el desarrollo del iPod del segundo.

Sin embargo el tamaño no es causa suficiente para innovar exitosamente. Se requiere un diseño apropiado en cuatro dimensiones: en primer lugar la estructura de la organización, no en términos de centralización-descentralización, sino de la "mezcla" adecuada para responder de manera ágil a las etapas tempranas del proceso de desarrollo, pero eficiente y controlada en las etapas de producción y distribución; segundo, en la dotación y desarrollo del capital humano, de las competencias y la calidad en la toma de decisiones en condiciones de racionalidad limitada; tercero, la conformación de una red de vínculos hacia el exterior de la empresa lo mismo para adquirir conocimiento como para producir los bienes o comercializarlos, básicamente por razones de diferencia entre los costos de transacción y los costos de administración; y finalmente, la cultura y los valores de la organización, la disposición para favorecer la prueba y el error, el permiso de experimentar, la comunicación, la confianza y la orientación al riesgo (Teece, 2000). En todo caso, cada uno de estos elementos puede estar sujeto a distintos arreglos para ser coordinado en el mercado o coordinado en la organización, dependiendo de los costos relativos de transacción, con excepción de los valores y la cultura del sistema cooperativo.

\section{La interpretación económica de la innovación en síntesis}

De la discusión previa se derivan las siguientes implicaciones. Primero, la innovación debe entenderse como un fenómeno económico y de ninguna otra manera (Say, 2002; Schumpeter, 2002, 2004; Solow, 1956; Romer, 1986), "personificada" en un producto, en una tarea o en un proceso para poder ser distinguida como tal (Boldrin y Levine, 2002, 2003), haciéndole una acto de naturaleza

ISSN: 07I 8-2724. (http://www.jotmi.org)

Journal of Technology Management \& Innovation @ Universidad Alberto Hurtado, Facultad de Economía y Negocios 
diferente al descubrimiento y a la invención. Segundo, y en consecuencia, aún cuando se pueden introducir nuevos productos, nuevas prácticas, nuevas tecnologías o nuevas formas de organización, sólo aquellas que producen riqueza transfiriendo "los recursos de campos de baja productividad y rendimiento a niveles de mayor productividad y rendimiento" (Drucker, 1988, p. 42) pueden ser consideradas como innovaciones. Tercero, el cambio tecnológico y la innovación ocurren como un evento endógeno y propio del sistema económico (Schumpeter, 2003; Romer, 1986). Cuarto, de acuerdo con los supuestos previos y la evidencia de diversos estudios, el cambio tecnológico y la innovación se difunden exitosamente en el mercado por la acción de la gran empresa y no por la acción del emprendedor y de la organización emprendedora (Mansfield, 1963; Henderson, 1993; Williamson, 1965, 1975, 1995; Teece, 2000). Por supuesto, el emprendedor tiene una función en el proceso de innovación como lo demuestra el desarrollo del formato MP3 (Winamp), pero la difusión a gran escala para crear valor económico ha estado más en manos de empresas como Apple (iPod), Sony (Aura) y Yahoo! Inc. (Musicmatch), siendo esta última un ejemplo en contrario para el sector de los "buscadores" de Internet. Cuando se crea enteramente una nueva categoría de productomercado, y sólo entonces, aumentan las posibilidades de la organización emprendedora para difundir la innovación y capturar mayoritariamente los incentivos de mercado (Teece, 2000; Gompers, 2002).

Aún cuando los autores de las diferentes perspectivas económicas revisadas coinciden en la definición básica de la innovación, no ocurre lo mismo con la definición operacional y la medición de la misma. Para algunos es la introducción visible de nuevos productos, de nuevos procesos, la creación de nuevos mercados, el diseño de nuevas formas de organización o el empleo de nuevos factores (Mansfield, 1963; Scherer, 1982; Geroski y Pomroy, 1990; Drucker, 1988, 1998; Henderson, 1993; Koeller, 1995; Corriveau, 1998; Gompers, 2002; More, 2000; Rosenberg, 2000; Schumpeter, 2002, 2004). Para otros la innovación se mide por el crecimiento de la economía, debido a distintas causas como el incremento en la fuerza laboral y en la tasa de ahorro e inversión (Brunn, 1974; Romer, 1990; Aghion, 2002; Schumpeter,
2002, 2004). Dada la orientación al cambio tecnológico, la innovación se ha evaluado también por el número de patentes producidas (Scherer, 1982; Aghion, 2002; Acemoglu y Linn, 2003) y finalmente, por los efectos en la estructura de mercado, dimensionándola por los cambios en la concentración y en la participación de las empresas individuales (Geroski y Pomroy, 1990; Robinson, 1990; Henderson, 1993; Afuah, 2002).

A partir de la definición operacional, las variables independientes pueden organizarse por su naturaleza en cuatro grandes grupos: primero, aquellas relacionadas con los estímulos de la innovación -sea la ganancia del emprendedor, los incentivos del mercado, la búsqueda de beneficios económicos- (Schumpeter, 1975, 2002, 2004; Romer, 1990; More, 2000; Rosenberg, 2000; Acemoglu y Linn, 2003), o bien el escape de la competencia y la obtención de ventajas competitivas (Williamson, 1965; Geroski, 1989; Robinson, 1990; Gatti, 1998; Blundell, Griffith y Van Reenen, 1999; Porter, 1999; Boldrin y Levine, 2003). Segundo, aquellas haciendo referencia al tamaño de la empresa innovadora -considerando por un lado los trabajos que proponen al emprendedor $y$ a la organización emprendedora como la responsable de introducir las innovaciones (Say, 2002; Schumpeter, 2002, 2004; Gompers, 2002), y por el otro los estudios sugiriendo a la gran organización como tal, dada la disponibilidad de recursos (Williamson, 1965, 1975, 1995; Schumpeter, 1975; Teece, 2000). Tercero, aquellas relacionadas con el régimen de apropiación -bien por medio de patentes, del secreto industrial y los derechos de autor, o bien por la facilidad o la dificultad de imitación y replicación- (Nelson, 1959; Ashton, 1975; Robinson, 1990; Casas y Luna, 1997; Teece, 2000; Aghion, 2002); y finalmente aquellas relacionadas con la producción, transferencia y aplicación del conocimiento -tales como la inversión en investigación y desarrollo, la proporción de científicos, ingenieros y personal preparado con relación al personal total, la capacidad de aprendizaje y desarrollo de nuevas habilidades, y la capacidad de transferir conocimientos de cualquier fuente disponible (Koeller, 1995; Gatti, 1998; Porter, 1999; Teece, 2000; Adams y Marcu, 2004). La relación entre las dimensiones y las variables independientes aquí comentadas se muestran en la figura $I$. 


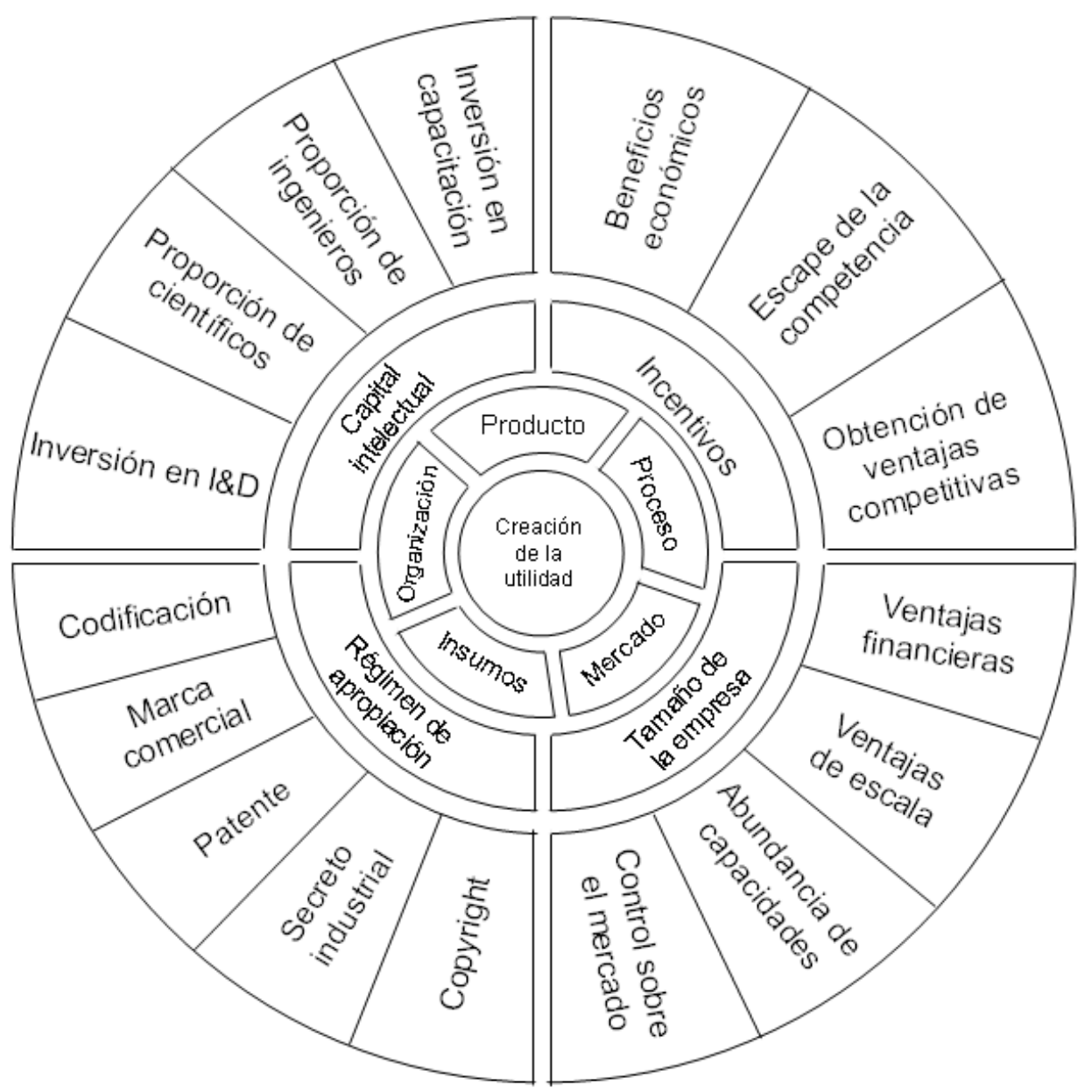

Figura I. Relación entre las dimensiones y las variables independientes en la interpretación de la disciplina económica sobre la innovación

\section{Conclusión}

La innovación es un fenómeno económico y organizacional de creación de valor que resulta de la nueva combinación de los factores de producción y del conocimiento, la cual se personaliza en la forma de un nuevo producto o servicio, de un nuevo método de producción, de un nuevo mercado o el uso de un nuevo insumo o de una nueva tecnología. De manera sencilla se diferencia de la ciencia y de la tecnología en tanto la primera es el descubrimiento de las leyes que explican un fenómeno y el segundo la invención. En algunos casos el agente impulsor de la innovación será el emprendedor, particularmente para el cambio disruptivo y en otras la organización empresarial establecida para la introducción de cambios sostenibles.
Una de las nociones de mayor relevancia para el entendimiento de la innovación derivada de la presente revisión se refiere al carácter particular del conocimiento, aquel factor con el potencial de alterar el concepto de los retornos decrecientes en la producción y la utilidad del consumo, por el concepto de los retornos crecientes en la actividad económica. La importancia del conocimiento ha sido discutida desde J.B. Say en el 1803, obligando a replantear los supuestos detrás de la teoría del equilibrio. Como lo expone Teece, "La innovación sólo se refiere a la creación y al uso del conocimiento” (2000, p. 35).

Pero para la teoría económica neoclásica, específicamente para la interpretación de la estructura industrial y la competencia en los mercados, el principio de los retornos

ISSN: 07I 8-2724. (http://www.jotmi.org)

Journal of Technology Management \& Innovation (C) Universidad Alberto Hurtado, Facultad de Economía y Negocios 
crecientes hace incompatible la noción de equilibrio entre la oferta y la demanda, un concepto central en la teoría tradicional: si los retornos en la producción no disminuyen en algún punto, entonces la oferta podría crecer indefinidamente, lo cual resulta un absurdo desde el método neoclásico. Si esto es así, entonces el mercado perfecto de los economistas no es sino un espejismo y en el mejor de los casos un estado de transición, tema al cual hemos de referirnos en otra entrega. Faltará analizar el impacto sobre la utilidad marginal en el consumo, pero en principio éste planteamiento pudiera sugerir, primero, la necesidad de una interpretación evolutiva y cambiante entre la oferta y la demanda, y de confirmarse, entonces aceptar que las estructuras monopólicas y oligopólicas son el resultado natural del proceso mismo de la economía y no estructuras imperfectas como hasta ahora han sido consideradas.

\section{Consideraciones}

Este artículo fue realizado bajo el apoyo de la cátedra de innovación tecnológica del Tecnológico de Monterrey Campus Ciudad de México.

\section{Bibliografía}

ACEMOGLU, D., Linn, J. (2003). Market size in innovation: theory and evidence from the pharmaceutical industry. Cambridge, MA: National Bureau of Economic Research Working Paper Series, No. 10038.

ACS, Z. J., Audretsch, D. B. (1988). Innovation in large and small firms: an empirical analysis. The American Economic Review, 78(4): 678-690.

ADAMS, J. D., Marcu, M. (2004). R\&D sourcing, joint ventures and innovation: a multiple indicators approach. Cambridge, MA: National Bureau of Economic Research Working Paper Series, No. 10474.

AFUAH, A. (2002). Mapping technological capabilities into product market and competitive advantage: the case of cholesterol drugs. Strategic Management Journal, 23(2): I7I179.

AGHION, P., Bloom, N., Blundell, R., Griffith, R., Howitt, P. (2002). Competition and innovation: an inverted $u$ relationship. Cambridge, MA: National Bureau of Economic Research Working Paper Series, No. 9269.
ANDREW, J. P., Sirkin, H. L. (2006). Payback: reaping the rewards of innovation. Harvard Business School Press, Boston, MA.

ASHTON, T. S. (1975). La revolución industrial I760-/830. Fondo de Cultura Económica, Mexico. (Trabajo original publicado en 1948).

BAGGS, J., de Bettignies, J. E. (2006). Product market competition and agency costs. Sauder School of Business Working Papers: I-36.

BAS, T. G., Amoros, E., Kunc, M. (2008). Innovation, entrepreneurship and clusters in Latin America natural resource: implication and future challenges. Journal of Technology Management \& Innovation, 3(4): 52-65.

BARTEL, A. P., Sicherman, N. (1999). Technological change and wages: an interindustry analysis. The Journal of Political Economy, 107(2), 285-325.

BASALLA, G. (1999). The evolution of technology. Cambridge University Press, Cambridge, UK. (Original work published 1988).

BEINHOCKER, E. D. (2006). The origin of wealth: evolution, complexity, and the radical remaking of economics. Harvard Business School Press, Boston, MA.

BLUNDELL, R., Griffith, R., Van Reenen, J. (1999). Market share, market value and innovation in a panel of British manufacturing firms. The Review of Economic Studies, 66(3): 529-554.

BOLDRIN, M., Levine, D. K. (2002). The theory of innovation without intellectual monopoly, California, LA: Levine's Working Paper Archives from UCLA Department of Economics.

BOLDRIN, M., Levine, D. E. (2003). Perfectly competitive innovation, National Science Foundation Papers, January 17.

BOLDRIN, M., Levine, D. K. (2004). Rent-seeking and innovation, Journal of Monetary Economics, 5I(I): 127-160.

BRUUN, G. (1974). La Europa del siglo XIX. Fondo de Cultura Económica, México. (Trabajo original publicado en 1959).

ISSN: 07/8-2724. (http://www.jotmi.org)

Journal of Technology Management \& Innovation @ Universidad Alberto Hurtado, Facultad de Economía y Negocios 
CASAS, R., Luna, M. (1997). Gobierno, academia y empresas en México. Plaza y Valdés Editores, México.

CHRISTENSEN, C. M. (2005). The innovator's dilemma. Harper Collins Publishers, New York, NY (Original work published 1997).

COASE, R. H. (1988). The firm, the market and the law. The University of Chicago Press, Chicago.

COASE, R. H. (1994). Essays on economics and economists. The University of Chicago Press, Chicago, IL.

CORRIVEAU, L. (1998). Innovation races, strategic externalities and endogenous growth. Economica, New Series, 65(259): 303-325.

DEMSETZ, H. (1997). The economics of the business firm: seven critical commentaries. Cambridge University Press, New York.

DRUCKER, P. F. (1988). La innovación y el empresariado innovador: la práctica y los principios. Hermes, México.

DRUCKER, P. F. (1998). On the profession of management. Harvard Business School Press, Boston, MA.

GATTI, D. (1998). Unemployment and innovation patterns: the role of business coordination and market competition, Wissenschaftszentrum Berlin für Sozialforschung: Discussion Paper FS I 98-306.

GEROSKI, P. A. (1989). Entry, innovation and productivity growth. The Review of Economic and Statistics, 7I(4): 572578.

GEROSKI, P. A., Pomroy, R. (1990). Innovation and the Evolution of Market Structure. The Journal of Industrial Economics, 38(3): 299-3।4.

GOMPERS, P. A. (2002). Corporations and the financing of innovation: the corporate venturing experience. Economic Review, 87(4), I-I7.

GOPALAKRISHNAN, S. (2000). Unraveling the links between dimensions of innovation and organizational performance. The Journal of High Technology Research Management, I I(I): I37-I53.

HENDERSON, R. (1993). Underinvestment and incompetence as responses to radical innovation: evidence

ISSN: 07| 8-2724. (http://www.jotmi.org)

Journal of Technology Management \& Innovation ( U Universidad Alberto Hurtado, Facultad de Economía y Negocios from photolithographic alignment equipment industry. The RAND Journal of Economics, 24(2): 248-270.

KOELLER, C. T. (1995). Innovation, market structure and firm size: a simultaneous equations model. Managerial and Decision Economics, 16(3), 259-269.

LASKI, H. J. (1977). El liberalismo europeo. Fondo de Cultura Económica, México (Trabajo original publicado en 1936).

LIENHARD, J. H. (2006). How invention begins: echoes of old voices in the rise of new machines. New York, NY: Oxford University Press.

LUCAS, R. (1993). Making a miracle. Econometrica, 6I(2), 25I-272.

MANSFIELD, E. (1963). Size of firm, market structure, and innovation. The Journal of Political Economy, 7 I (6): 556-576.

MORE, C. (2000). Understanding the Industrial Revolution. Routledge, London.

MOSER, P. (2003). How do patent laws influence innovation?: evidence from nineteenth-century world fairs. Cambridge, MA: National Bureau of Economic Research Working Paper Series, No. 9909.

NELSON, R. R. (1959). The simple economics of basic scientific research. The Journal of Political Economy, 67(3), 297-306.

OGDEN A. H., Teece, D. J. (1980). Vertical integration and technological innovation. The Review of Economics and Statistics, 62(3): 470-474.

ORDÓÑEZ, J. (200I). Ciencia, tecnología e historia: relaciones y diferencias. ITESM-Ariel, México.

PORTER, M. E. (1999). Ser competitivo: nuevas aportaciones y conclusiones. Editorial Deusto, España.

ROBINSON, W. T. (1990). Product innovation and startup business market share performance. Management Science, 36(10): 1279-1289.

ROMER, P. M. (1986). Increasing returns and long-run growth. The Journal of Political Economy, 94(5), 1002-1037. 
ROMER, P. M. (1990). Endogenous technological change. The Journal of Political Economy, 98(5), 7I-I02.

ROMO, D., Hill de Titto, P. (2006). Los determinantes de las actividades tecnológicas en México. Documentos de Trabajo en Ciencia y Tecnología del Centro de Investigación y Docencia Económicas, CIDECyT 06-0I, México.

ROSENBERG, N. (2000). Schumpeter and the endogeneity of technology. Routledge, London.

SAY, J. B. (2002). A treatise on political economy: or the production, distribution, and consumption of wealth. Batoche Books, Kitchener, ON.

SCHERER, F. M. (1982). Demand-pull and technological invention: Schmookler revisited. The Journal of Industrial Economics, 30(3), 225-237.

SCHUMPETER, J. A. (1975). Capitalism, socialism, and democracy. Harper Perennial, New York. (Original work published 1942).

SCHUMPETER, J. A. (2002). Essays: on entrepreneurs, innovations, business cycle, and the evolution of capitalism. Transaction Publishers, New Brunswick, NJ.

SCHUMPETER, J. A. (2004). The theory of economic development. Transaction Publishers, New Brunswick: (Original work published 1934).

SOLOW, R. M. (1956). A contribution to the theory of economic growth. Quarterly Journal of Economics, 70(I): 6594.

TEECE, D. J. (1986). Profiting from technological innovation: implications for integration, collaboration, licensing and public policy. Research Policy, No. 15: 285-305.

TEECE, D. J. (2000). Managing intellectual capital. Oxford University Press, New York:

UTTERBACK, J. M. (197I). The process of technological innovation within the firm. The Academy of management Journal, I4(I): 75-88.

UTTERBACK, J. M. (1996). Mastering the dynamics of innovation. Harvard Business School Press, Boston, MA. (Original work published 1994).
VARIAN, H. R. (2003). Intermediate microeconomics: a modern approach. W. W. Norton \& Co., New York, NY.

WILLIAMSON, O. E. (1965). Innovation and market structure, The Journal of Political Economy, 73(I): 67-73.

WILLIAMSON, O. E. (1975). Markets and hierarchies: analysis and antitrust implications. The Free Press, New York.

WILLIAMSON, O.E. (1985). The economic institutions of capitalism. The Free Press, New York.

WILLIAMSON, O. E. (1995). Transaction costs economics and organization theory. In O. Williamson (Ed.), Organization theory: from Chester Barnard to the present and beyond (pp. 207-256). Oxford University Press, New York.

ZAWISLAK, P. A., Marins, L. M. (2007). Strengthening innovation in developing countries, Journal of Technology Management \& Innovation, 2(4): 44-54. 